\title{
Clinical Study \\ Effect of an Oral Adsorbent, AST-120, on Dialysis Initiation and Survival in Patients with Chronic Kidney Disease
}

\author{
Shingo Hatakeyama, ${ }^{1}$ Hayato Yamamoto, ${ }^{2}$ Akiko Okamoto, ${ }^{3}$ Kengo Imanishi, ${ }^{3}$ \\ Noriko Tokui, ${ }^{2}$ Teppei Okamoto, ${ }^{2}$ Yuichiro Suzuki, ${ }^{2}$ Naoaki Sugiyama, ${ }^{2}$ \\ Atsushi Imai, ${ }^{2}$ Shigemasa Kudo, ${ }^{2}$ Takahiro Yoneyama, ${ }^{2}$ Yasuhiro Hashimoto, ${ }^{3}$ \\ Takuya Koie, ${ }^{2}$ Noritaka Kaminura, ${ }^{2}$ Hisao Saitoh, ${ }^{3}$ Tomihisa Funyu, ${ }^{3}$ and Chikara Ohyama ${ }^{1,2}$ \\ ${ }^{1}$ Department of Advanced Transplant and Regenerative Medicine, Hirosaki University Graduate School of Medicine, \\ 5 Zaifu-chou, Hirosaki 036-8562, Japan \\ ${ }^{2}$ Department of Urology, Hirosaki University Graduate School of Medicine, Hirosaki 036-8562, Japan \\ ${ }^{3}$ Department of Urology, Oyokyo Kidney Research Institute, Hirosaki 036-8243, Japan
}

Correspondence should be addressed to Chikara Ohyama, coyama@cc.hirosaki-u.ac.jp

Received 5 August 2011; Revised 23 September 2011; Accepted 5 October 2011

Academic Editor: Jochen Reiser

Copyright ( 2012 Shingo Hatakeyama et al. This is an open access article distributed under the Creative Commons Attribution License, which permits unrestricted use, distribution, and reproduction in any medium, provided the original work is properly cited.

\begin{abstract}
The oral adsorbent AST-120 has the potential to delay dialysis initiation and improve survival of patients on dialysis. We evaluated the effect of AST-120 on dialysis initiation and its potential to improve survival in patients with chronic kidney disease. The present retrospective pair-matched study included 560 patients, grouped according to whether or not they received AST-120 before dialysis (AST-120 and non-AST-120 groups). The cumulative dialysis initiation free rate and survival rate were compared by the KaplanMeier method. Multivariate analysis was used to determine the impact of AST-120 on dialysis initiation. Our results showed significant differences in the 12 - and 24 -month dialysis initiation free rate $(P<0.001)$, although no significant difference was observed in the survival rate between the two groups. In conclusion, AST-120 delays dialysis initiation in chronic kidney disease (CKD) patients but has no effect on survival. AST-120 is an effective therapy for delaying the progression of CKD.
\end{abstract}

\section{Introduction}

The number of dialysis patients is increasing worldwide [1] as a consequence of treatment to prevent progression of chronic kidney disease (CKD) remaining largely unresolved. To prevent CKD progression from an early stage, many clinical studies have suggested that disease progression may be curbed by controlling dietary factors, blood pressure, lipids, anemia, and mineral balance [2]. However, management of protein and salt restriction along with blood pressure is not always effective in patients using a multidisciplinary approach, including the use of angiotensin-converting enzyme inhibitors (ACEIs) and angiotensin receptor blockers (ARBs), that forms the basic strategy currently used to control CKD progression $[1,3]$. This suggests the necessity for further development of modalities to control CKD progression.
AST-120 (Kremezin, Kureha Corporation, Tokyo, Japan) is an oral adsorbent consisting of microspheres made from porous carbon material [4]. By adsorbing uremic toxins, including indoxyl sulfate, AST-120 can slow CKD progression and delay dialysis initiation. The drug has been clinically used in Japan since 1991 and is covered by health insurance $[5,6]$.

Although large-scale multicenter trials have shown the effect of AST-120 to delay dialysis initiation in CDK patients, these reports were published in the late 1980s [7]. Updating data on the AST-120 efficacy for delaying dialysis initiation is therefore necessary under the currently employed basic strategy. Recently, Ueda et al. [8] and Maeda et al. [9] reported the efficacy of AST-120 on CKD progression and delay in dialysis initiation. These studies used the propensity score matching method to compare the efficacy of AST-120, although the number of patients studied was relatively small. 
The importance of early diagnosis and treatment of CKD patients is well established as cardiovascular disease is known to be the primary cause of high mortality in these patients. Recently, Ueda et al. [10] reported improved prognosis in CKD patients who had been administered AST-120 during the predialysis period. However, further evidence is required to elucidate the effect of AST-120 on the prognosis of dialysis patients [11].

In the present study, we performed a retrospective examination on the therapeutic effect of AST-120 in combination with current basic treatment regimens in CKD patients. To guarantee the validity of the retrospective analysis we used the propensity score method to identify a matched-control group.

\section{Patients and Methods}

Between January 1991 and December 2010, 872 of CKD patients were followed until dialysis initiation at Oyokyo Kidney Research Institute, Hirosaki. Of the 872 patients, 363 had a history of using AST-120, and 509 patients had never received AST-120. By applying the propensity score matching method, 560 patients were pair-matched ( $n=280$ of each group) and enrolled in the study. Dialysis initiation was determined based on scores consisting of (1) clinical symptom (fluid retention, electrolyte abnormality, gastrointestinal symptoms, circulatory symptoms, neurological symptoms, hematological disorders, and vision disorders), (2) remnant kidney function (serum creatinine or creatinine clearance), (3) impairment level of daily living, (4) age (10 years or less, and 65 or older), and (5) existence of systemic vascular disorder according to the guidelines for introducing patients to dialysis issued by the Ministry of Health, Labour and Welfare of Japan. Patients with sum total 60 or higher were indication for dialysis initiation (Table 1) [12]. This study was approved by the institutional ethical committee of Oyokyo Kidney Research Institute.

2.1. Pair-Matching Methods. To guarantee the validity of this retrospective analysis, a propensity score was applied to pairmatched patients. Propensity scores were calculated using logistic analysis. The data used in the analyses included age, gender, blood pressure, biochemistry, concomitant drugs (activated vitamin-D, ACEIs, ARBs, and calcium blockers), estimated glomerular filtration rate (eGFR), presence of diabetes mellitus, and cardiovascular disease (heart failure, myocardial infarction and angina pectoris) at the initial visit. The eGFR was calculated using age, gender, and serum creatinine $(\mathrm{sCr})$ by the equation shown below [13]. This eGFR equation for Japanese patients is a modified version of the abbreviated Modification of Diet in Renal Disease Study formula [14]. [eGFR $\mathrm{mL} / \mathrm{min} / 1.73 \mathrm{~m}^{2}=194 \times \mathrm{sCr}^{-1.094} \times$ age $^{-0.287}(\times 0.739$, if female $\left.)\right]$. AST-120 administration quitted after the initiation of hemodialysis.

Based on the scores of each patient, two patients with a score within 0.03 were selected as a pair. For the AST-120 group, the baseline was defined as the time of initiation of AST-120 treatment. Baseline for patients in the control group was defined as the date of measurement of eGFR that was closest to the baseline eGFR level of their counterpart in the AST-120 group.

We compared the effect of AST-120 to delay dialysis initiation in patients taking AST-120 (AST-120 group) with pair-matched patients not taking AST-120 (non-AST-120 group). Because all patients received dialysis, the 12- and 24month dialysis initiation free rate was assessed as primary endpoints between the pair-matched AST-120-treated and non-AST-120 treated groups. The 3-, 5-, and 10-year survival rate after administration of AST-120 was assessed as a secondary endpoint.

2.2. Evaluation. The background clinical data and concomitant drugs of the two groups were compared using the chisquare test. Age and other biochemical parameters were expressed as mean $\pm \mathrm{SD}$, and statistical differences were calculated by Student's $t$-test. The level of urinary protein excretion was expressed as median and tested by MannWhitney's $U$-test. Cumulative dialysis initiation free rate and survival rate were plotted by the Kaplan-Meier method, and intergroup differences were tested by the log-rank test. A $P$ value of less than 0.05 was considered significant. Cox regression adjusted for these factors was also performed. All analyses were performed using SPSS (SPSS Inc, ver. 12.0, Chicago, IL, USA).

\section{Results}

The baseline characteristics of the matched patients are summarized in Table 2. After matching, no significant differences were observed in patients' background between the two groups. At the time of dialysis initiation, the effect of AST-120 on blood pressure, blood, and serum data were compared between AST-120 and non-AST-120 group (Table 3). There was no difference in blood and serum data but systolic and diastolic blood pressure showed significant difference between two groups $(P=0.0217$ and $P=0.0180$, resp.)

The effect of AST-120 on dialysis initiation in the pairmatched patients is shown in Figure 1. The 12- and 24month dialysis initiation free rate was significantly higher in the AST-120 group (25.0\% and $13.7 \%$, resp.) than in the non-AST-120 group (10.5\% and 5.7\%, resp.) (Figure 1, Table 4).

Using subgroup analysis, we evaluated the efficacy of AST-120 in patients with diabetic nephropathy or nondiabetic renal disease, and also with or without cardiovascular disease. For patients with diabetic nephropathy or cardiovascular disease, the 12- and 24-month dialysis initiation free rate was significantly higher in the AST-120 group than in the non-AST-120 group (Figure 2, Table 4). This delay in dialysis initiation was longer in patients with cardiovascular disease or those without diabetic nephropathy.

We performed a Cox's proportional hazard model based on uni- and multivariate analysis to determine the independent factors for dialysis initiation in the pair-matched patients. For univariate analysis we selected eGFR and not 
TABLE 1: Dialysis initiation criteria in Japan. According to the guidelines for introducing patients to dialysis issued by the Ministry of Health, Labour and Welfare of Japan, sum total of 60 or higher is indication for dialysis initiation.

\begin{tabular}{|c|c|c|}
\hline Items & & Score \\
\hline \multirow{10}{*}{ Clinical symptoms } & Fluid retention & \\
\hline & Electrolyte abnormality & \\
\hline & Gastrointestinal symptoms & \\
\hline & Circulatory symptoms & \\
\hline & Neurological symptoms & \\
\hline & Hematological disorders & \\
\hline & Vision disorders & \\
\hline & High grade & 30 \\
\hline & Intermediate grade & 20 \\
\hline & Low grade & 10 \\
\hline \multirow{5}{*}{ Remnant Renal function } & serum creatinine $(\mathrm{mg} / \mathrm{d})$ & \\
\hline & (creatinine clearance: $\mathrm{CCr}(\mathrm{mL} / \mathrm{min}))$ & \\
\hline & $\mathrm{sCr} 8 \mathrm{mg} / \mathrm{mL}$ or higher $(\mathrm{CCr}<10 \mathrm{~mL} / \mathrm{min})$ & 30 \\
\hline & $\mathrm{sCr} 5 \sim 8 \mathrm{mg} / \mathrm{mL}(\mathrm{CCr} 10 \sim 20 \mathrm{~mL} / \mathrm{min})$ & 20 \\
\hline & $\mathrm{sCr} 3 \sim 5 \mathrm{mg} / \mathrm{mL}(\mathrm{CCr} 20 \sim 30 \mathrm{~mL} / \mathrm{min})$ & 10 \\
\hline \multirow{3}{*}{ Impairment level of daily living } & High grade: impossible to wake-up & 30 \\
\hline & Intermediate grade: marked limitation of activity & 20 \\
\hline & Low grade: slight limitation of activity & 10 \\
\hline \multirow{2}{*}{ Age } & 10 years or less & 10 \\
\hline & 65 years or older & 10 \\
\hline Systemic vascular disorder & Existence of systemic vascular disorder & 10 \\
\hline
\end{tabular}

TABLE 2: Characteristics of 560 pair-matched patients. No significant differences were observed in the backgrounds of the two groups. CKD: chronic kidney disease, BMI: body mass index, Hb: hemoglobin, eGFR: estimated glomerular filtration rate, Alb: albumin, IP: inorganic phosphate, Ca: calcium, IP: inorganic phosphorus, BP: blood pressure, CVD: cardiovascular disease, DM: diabetes mellitus, Vit D: vitamin D, ACEIs: angiotensin-converting enzyme inhibitors, ARBs: angiotensin receptor blockers.

\begin{tabular}{|c|c|c|c|c|}
\hline & All & non-AST-120 & AST-120 & $P$ value \\
\hline$n$ & 560 & 280 & 280 & \\
\hline Age & $66.2 \pm 12.3$ & $66.4 \pm 12.0$ & $66.1 \pm 12.6$ & 0.7869 \\
\hline Gender $(\mathrm{M} / \mathrm{F})$ & $345 / 215$ & $181 / 99$ & $164 / 116$ & 0.1396 \\
\hline \multicolumn{5}{|l|}{ CKD stage, $n(\%)$} \\
\hline 3 & $4(0.7)$ & $3(1.1)$ & $1(0.4)$ & \\
\hline 4 & $19(3.4)$ & $14(5.0)$ & $15(5.3)$ & 0.5956 \\
\hline 5 & $527(94.0)$ & $263(93.9)$ & $264(94.3)$ & \\
\hline \multicolumn{5}{|l|}{ Baseline data } \\
\hline BMI & $23.7 \pm 4.1$ & $23.7 \pm 4.2$ & $23.7 \pm 4.0$ & 0.8642 \\
\hline $\mathrm{Hb}(\mathrm{g} / \mathrm{dL})$ & $8.8 \pm 1.7$ & $8.9 \pm 1.8$ & $8.8 \pm 1.6$ & 0.6265 \\
\hline eGFR (mL/min) & $8.0 \pm 5.7$ & $8.0 \pm 6.9$ & $8.0 \pm 4.2$ & 0.8847 \\
\hline Alb (g/dL) & $3.6 \pm 0.6$ & $3.6 \pm 0.6$ & $3.6 \pm 0.6$ & 0.9107 \\
\hline IP (mg/dL) & $5.4 \pm 1.5$ & $5.4 \pm 1.6$ & $5.5 \pm 1.5$ & 0.8662 \\
\hline Corrected $\mathrm{Ca}(\mathrm{mg} / \mathrm{dL})$ & $8.4 \pm 1.1$ & $8.4 \pm 1.0$ & $8.4 \pm 1.1$ & 0.9841 \\
\hline Systolic BP (mmHg) & $160 \pm 27.3$ & $160 \pm 27.7$ & $159 \pm 27.0$ & 0.706 \\
\hline $\operatorname{CVD}(-/+), n$ & $289 / 271$ & $144 / 136$ & $145 / 135$ & 0.9326 \\
\hline $\mathrm{DM}(-/+), n$ & $282 / 278$ & $137 / 143$ & $145 / 135$ & 0.4989 \\
\hline Use of activated Vit $\mathrm{D}(-/+), n$ & $172 / 388$ & $88 / 192$ & $84 / 196$ & 0.714 \\
\hline Use of ACEls/ARBs $(-/+), n$ & $182 / 378$ & $92 / 188$ & $90 / 190$ & 0.8568 \\
\hline Use of Ca Blocker $(-/+), n$ & $73 / 478$ & $36 / 244$ & $37 / 243$ & 0.9001 \\
\hline Urine protein $(\mathrm{mg} / \mathrm{dL})$ (median) & 264 & 200 & 270 & 0.2842 \\
\hline
\end{tabular}


TABLE 3: The effect of AST-120 on blood pressure, blood, and serum data at the time of dialysis initiation.

\begin{tabular}{|c|c|c|c|c|}
\hline At the time of HD initiation & All & non AST-120 & AST-120 & $P$ value \\
\hline $\mathrm{Hb}(\mathrm{g} / \mathrm{L})$ & $8.6 \pm 1.5$ & $8.5 \pm 1.6$ & $8.7 \pm 1.5$ & 0.1276 \\
\hline BUN (mg/dL) & $96.6 \pm 29.9$ & $94.4 \pm 28.6$ & $98.6 \pm 31.1$ & 0.1786 \\
\hline Serum creatinine $(\mathrm{mg} / \mathrm{dL})$ & $8.7 \pm 4.8$ & $8.4 \pm 2.8$ & $9.0 \pm 6.1$ & 0.1702 \\
\hline IP $(\mathrm{mg} / \mathrm{dL})$ & $6.2 \pm 1.6$ & $6.1 \pm 1.6$ & $6.3 \pm 1.6$ & 0.4590 \\
\hline Total protein $(\mathrm{g} / \mathrm{dL})$ & $6.1 \pm 0.8$ & $6.1 \pm 0.9$ & $6.1 \pm 0.8$ & 0.6722 \\
\hline Albumin (g/dL) & $3.4 \pm 0.6$ & $3.4 \pm 0.6$ & $3.4 \pm 0.6$ & 0.5151 \\
\hline Corrected Ca (mg/dL) & $8.5 \pm 1.1$ & $8.5 \pm 1.1$ & $8.6 \pm 1.1$ & 0.6528 \\
\hline Systolic BP (mmHg) & $162 \pm 25.5$ & $165 \pm 26.5$ & $159 \pm 24.2$ & 0.0217 \\
\hline Diastolic BP (mmHg) & $83.2 \pm 13.8$ & $85.0 \pm 14.1$ & $81.5 \pm 13.3$ & 0.0180 \\
\hline
\end{tabular}

TABle 4: The 12- and 24-month dialysis initiation free rate. The dialysis initiation free rate was significantly higher in the AST-120 group in all the pair-matched patients and also subgroups with or without DM or CVD.

\begin{tabular}{|c|c|c|c|}
\hline Dialysis initiation free rate (\%) & 12 months & 24 months & $P$ value \\
\hline AST-120 & 25.0 & 13.7 & \multirow{2}{*}{$<0.0001$} \\
\hline non-AST-120 & 10.5 & 5.7 & \\
\hline AST-120, DM(-) & 30.1 & 17.6 & \multirow{2}{*}{$<0.0001$} \\
\hline non-AST-120, DM(-) & 11.2 & 8.2 & \\
\hline AST-120, DM(+) & 20.8 & 10.0 & \multirow{2}{*}{0.0057} \\
\hline non-AST-120, DM(+) & 10.8 & 3.6 & \\
\hline $\operatorname{AST}-120, \operatorname{CVD}(-)$ & 24.2 & 22.6 & \multirow{3}{*}{0.0063} \\
\hline non-AST-120, CVD(-) & 11.2 & 8.4 & \\
\hline $\operatorname{AST}-120, \mathrm{CVD}(+)$ & 27.2 & 12.8 & \\
\hline non-AST-120, CVD $(+)$ & 9.8 & 2.9 & $<0.0001$ \\
\hline
\end{tabular}

TABLE 5: Uni- and multivariate analyses of the delaying effect of AST-120 on dialysis initiation. Uni- and multivariate analyses showed anemic status, eGFR, and not receiving AST-120 medication as independent factors associated with a significant increase in risk of dialysis initiation.

\begin{tabular}{|c|c|c|c|}
\hline Univariate analysis & $P$ value & Hazard ratio & $95 \%$ CI \\
\hline Age, $<66.2$, versus $\geq 66.2$ & 0.808 & 0.979 & $0.828-1.158$ \\
\hline CVD, without versus with & 0.883 & 1.013 & $0.828-1.158$ \\
\hline DM, without versus with & 0.918 & 1.009 & $0.854-1.191$ \\
\hline Systolic BP, $<140$, versus $\geq 140$ & 0.228 & 1.123 & $0.930-1.355$ \\
\hline BMI, $<22$, versus $\geq 22$ & 0.960 & 1.004 & $0.846-1.193$ \\
\hline ACEls/ARBs, without versus with & 0.778 & 1.026 & $0.859-1.225$ \\
\hline Ca blocker, without versus with & 0.414 & 0.902 & $0.705-1.155$ \\
\hline Activated Vit-D, without versus with & 0.725 & 0.968 & $0.809-1.159$ \\
\hline $\mathrm{Hb},<8.8$, versus $\geq 8.8$ & 0.064 & 0.853 & $0.721-1.010$ \\
\hline Alb, $<3.6$ versus, $\geq 3.6$ & 0.996 & 1.000 & $0.846-1.183$ \\
\hline Corrected $\mathrm{Ca},<8.4$ versus $\geq 8.4$ & 0.681 & 1.036 & $0.875-1.227$ \\
\hline Urine protein $(\mathrm{mg} / \mathrm{dL})$ & 0.087 & 1.000 & $1.000-1.001$ \\
\hline Urine protein (g/day) & 0.488 & 1.001 & $0.999-1.003$ \\
\hline eGFR, $<8.0$, versus $\geq 8.0$ & 0.007 & 0.784 & $0.657-0.937$ \\
\hline AST-120, with versus without & 0.000 & 1.812 & $1.530-2.145$ \\
\hline \multicolumn{4}{|l|}{ Multivariate analysis } \\
\hline $\mathrm{Hb},<8.8$, versus $\geq 8.8$ & 0.042 & 0.837 & $0.705-0.994$ \\
\hline eGFR, $<8.0$, versus $\geq 8.0$ & 0.005 & 0.773 & $0.645-0.925$ \\
\hline AST-120, with versus without & 0.000 & 1.881 & $1.586-2.231$ \\
\hline
\end{tabular}


TABLE 6: The 3-, 5-, and 10-year survival rate of pair-matched patients. The 3-, 5-, and 10-year survival rates from administration of AST-120 were analyzed by the Kaplan-Meier method with a log-rank test. Survival rates at 3-, 5-, and 10-years were not significantly different in the AST-120 and non-AST-120 groups.

\begin{tabular}{lcccc}
\hline Survival & 3-year & 5-year & 10-year & $P$ value \\
\hline AST-120 & 79.0 & 63.8 & 29.8 & 0.0664 \\
non-AST-120 & 78.1 & 65.6 & 44.0 \\
\hline
\end{tabular}

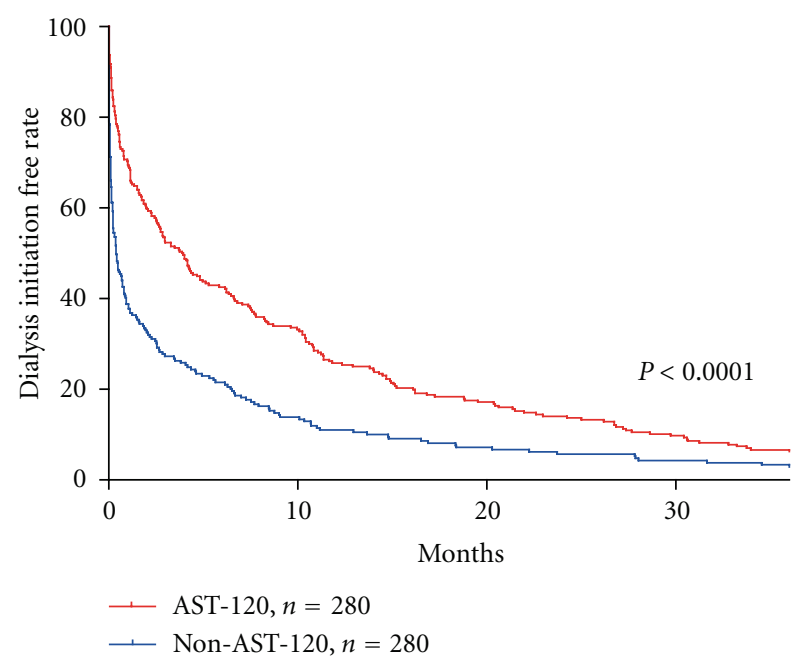

FIGURE 1: The delaying effect of AST-120 on dialysis initiation. The cumulative percentage of nondialysis patients analyzed by KaplanMeier methods showed the delaying effect of AST-120. The dialysis initiation free rate was significantly higher in the pair matched patients receiving AST-120 (AST-120 group, $n=280$ ) compared to those not receiving AST-120 (non-AST-120 group, $n=280$ ) $(P<0.001)$.

receiving AST-120 as independent factors for increasing the risk for dialysis initiation. Similarly, for multivariate analysis we selected anemic status, eGFR, and not receiving AST-120 as independent factors associated with a significant increase in risk of dialysis initiation (Table 5).

To examine whether or not the use of AST-120 in the predialysis period influenced prognosis after dialysis initiation, we compared the 3-, 5-, and 10-year survival rate from administration of AST-120 by the Kaplan-Meier method with a log-rank test. We found that there was no significant difference in survival rates between the AST-120 and nonAST-120 groups $(P=0.0664)$ (Table 6, Figure 3 ).

\section{Discussion}

CKD is recognized as a disease that increases the risk of many adverse events closely associated with death, such as cardiovascular disease [15]. Early diagnosis and treatment are necessary to delay CKD and prevent these adverse events. AST-120 is an oral adsorbent that slows the progression of CKD by decreasing serum nephrotoxic substances such as indoxyl sulfate. Recently, the CAP-KD study was conducted to evaluate the usefulness of AST-120 in patients with moderate to severe CKD [16]. This study showed no significant difference in the composite primary endpoints (doubling of sCr level, increase in $\mathrm{sCr}$ level $\geq 6.0 \mathrm{mg} / \mathrm{dL}$, need for dialysis or transplantation, and death), but revealed a significant suppression in the decrease of estimated GFR over 56 weeks in the AST-120 group. However, because of the short follow-up period, the CAP-KD study could not clarify the impact of AST-120 on the development of end-stage renal disease [16].

In the present study, we retrospectively analyzed the effects of AST-120 to delay dialysis initiation and survival in the context of current treatment regimens. Our results showed a significantly lower rate of dialysis initiation in the AST-120 group. This indicated that AST-120 is a useful treatment and supports recent retrospective studies by Ueda et al. [8] and Maeda et al. [9] that also showed AST-120 delayed dialysis initiation. Furthermore, Maeda et al. [17] reported long-term treatment of chronic renal failure with AST-120 from early CKD stage has potential to slowing progression of renal failure and delaying initiation of dialysis. In this present study, our result has limitation because main patients were at the late stage of CKD (CKD stage 5) and half of patients are initiated to hemodialysis within half year. These results suggest that AST-120 administration from early CKD stage has potential to improve the progression of renal failure effectively.

The present study had some limitations. Although the pair-matching method minimizes imbalances in patient background, the finding that AST-120 delayed dialysis initiation is not definitive, given that this was a retrospective study on a relatively small number of patients from a single institute. It is possible that the better outcomes in the AST120 group may be due to selection bias, and therefore a prospective, randomized trial with long-term followup is essential to evaluate the effectiveness of AST-120. In addition, we could not evaluate the effects of AST-120 on blood and serum data from AST-120 administration to dialysis initiation. Our data showed significant decrease in blood pressures at the time of dialysis initiation, but we could not conclude the effect of AST-120 on blood pressures because of retrospective limitations. Recently, Nakamura et al. [18] published AST-120 effects on sCr, eGFR, serum interleukin6 (IL-6), proteinuria, and urinary excretion levels of 8hydoxydeoxyguanosine (8-OHdG) and L-fatty acid binding protein (L-FABP), markers of oxidative stress and tubular injury. In their prospective study, 50 patients were divided in to 2 groups (AST-120 and non-AST-120 group, $n=25$ each) and followed up for 12 months. They showed AST-120 treatment significantly reduced sCr, eGFR, IL-6, proteinuria 8-OHdG, and L-FABP, but no effect on blood pressure. This 


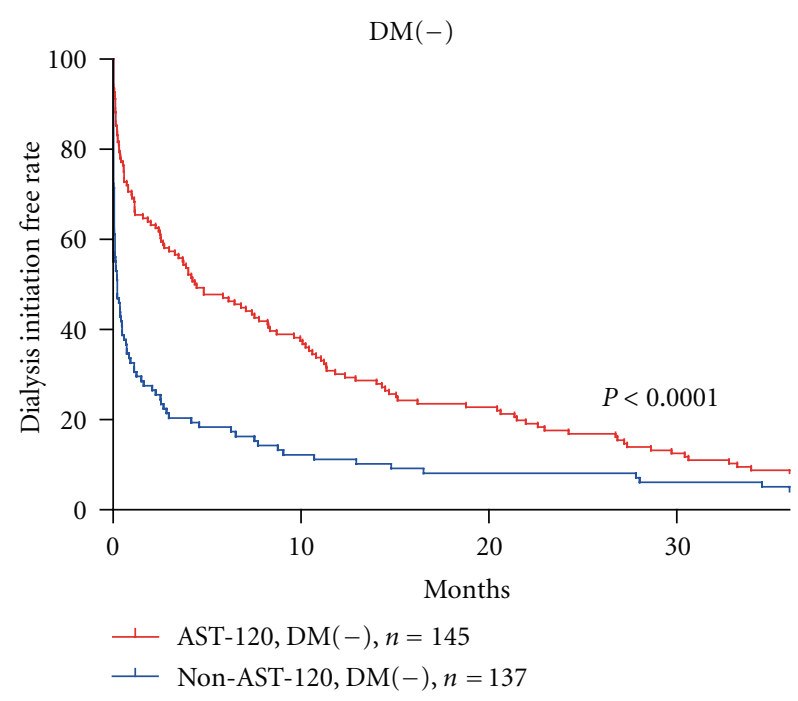

(a)

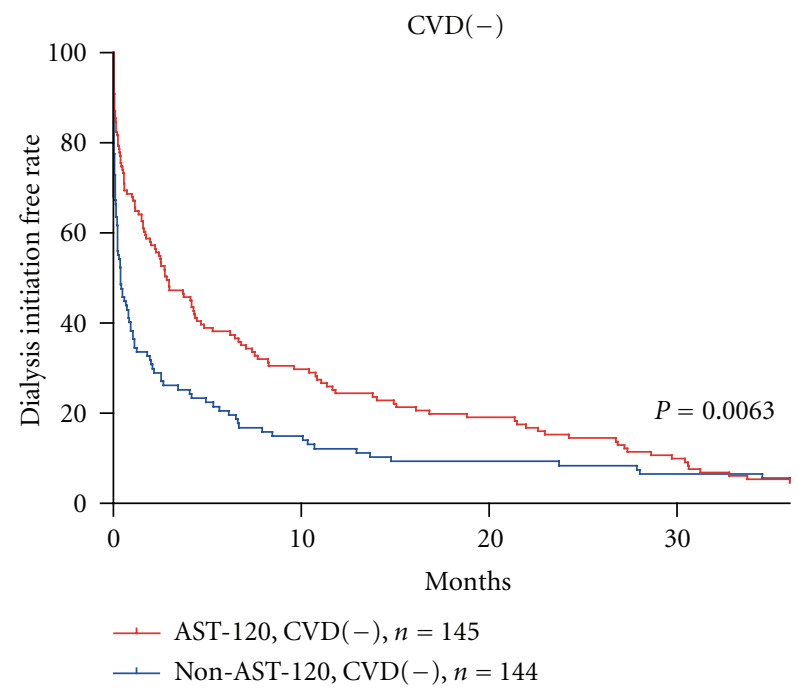

(c)

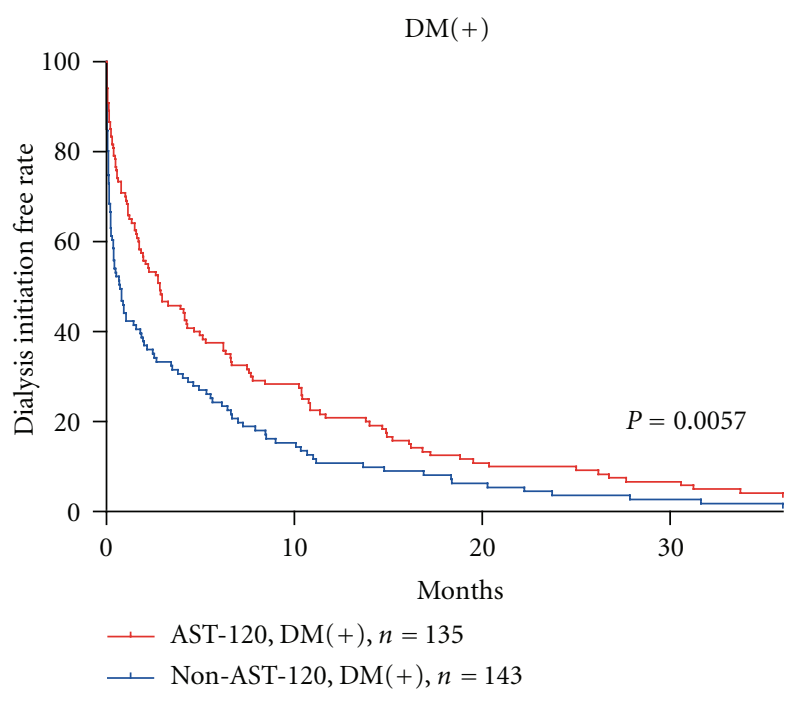

(b)

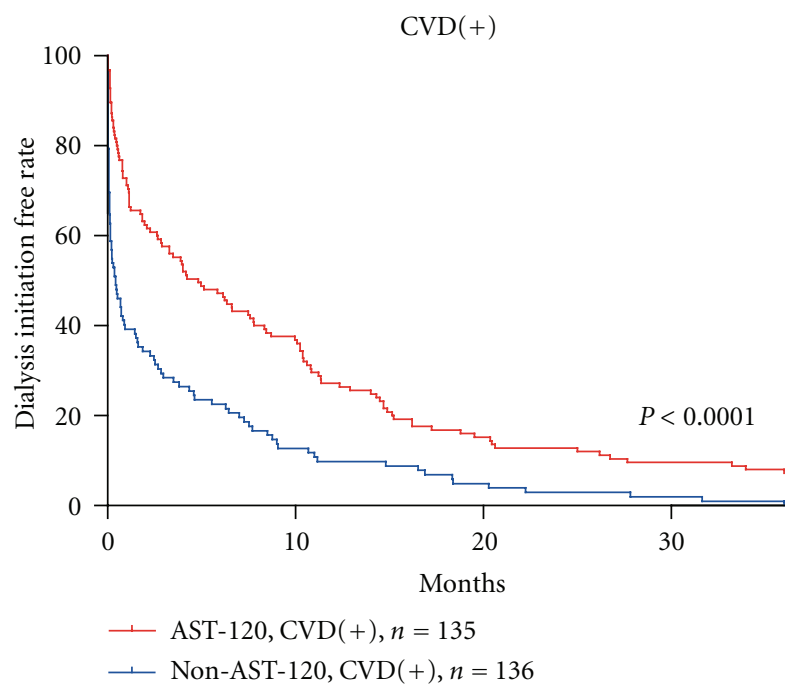

(d)

FIGURE 2: Subgroup analysis of the delaying effect of AST-120 on dialysis initiation. The cumulative percentages of non-dialysis patients were analyzed by Kaplan-Meier methods. The dialysis initiation free rate was significantly higher in the AST-120 group than in the non-AST-120 group in all subgroups, with DM [11] (a) or without DM (b), and with CVD (c) or without CVD (d).

study suggests AST-120 has potential to protect kidney from stresses and injury.

In addition to its known effect of delaying dialysis initiation, the influence of AST-120 on subsequent prognosis requires further study. Recent studies suggested that high levels of serum indoxyl sulfate, one of the substances known to enhance progression of atherosclerotic lesions, increase the risk of a cardiovascular event or death in dialysis patients [19]. These studies showed an association between the serum levels of indoxyl sulfate and mortality in CKD patients, indicating that this nephrotoxic compound may be involved in CKD progression and vascular disease [19]. Ueda et al. [10] suggested that patients receiving AST-120 before dialysis initiation tend to have an improved prognosis. In their report, the 5-year survival rate was significantly higher in the AST-120 group (72.6\% in the AST- 120 group and $52.6 \%$ in the non-AST-120 group). However, our results showed no significant difference in survival rates between the pairmatched AST-120 and non-AST-120 groups. This conflict result might be caused by the initial CKD stage difference in AST-120 administration. In the present study, 94\% of patients were stage $5(\mathrm{eGFR}, 8.0 \pm 5.7 \mathrm{~mL} / \mathrm{min})$ but CKD stage of study population in Ueda et al. [10] was stage 4 (average of eGFR, 24-25 mL/min). Yu et al. [20] prospectively investigated the role of indoxyl sulfate in endothelial dysfunction in CKD stage 4 patients and reported that AST-120 administration improved endothelial dysfunction associated with a decrease in indoxyl sulfate and a restoration 


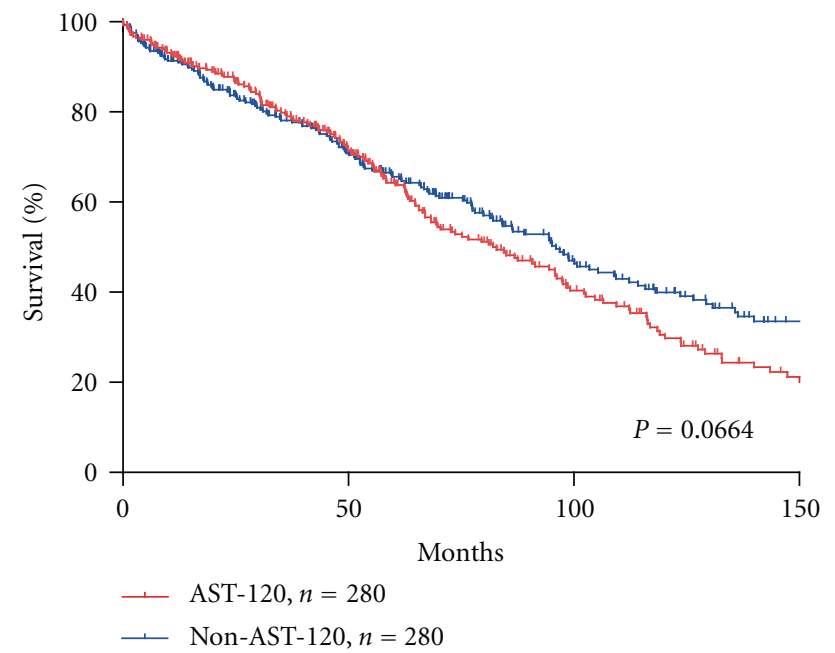

FIgure 3: Patient survival rate after the administration of AST120. Patient survival rate was not significantly different in the pairmatched patients with or without AST-120 treatment.

of antioxidant reserve. H. Shibahara and N. Shibahara [21] reported AST-120 administration contributed to the improvement of cardiac and renal function in moderate CKD patients (sCr, $1.3-2.0 \mathrm{mg} / \mathrm{dL}$ ) in their prospective study. In addition, Nakamura et al. [18] evaluated AST-120 effects for tubular damage through the reduction of proteinuria and oxidative stress generation in CKD stage 4 patients. From these observations, we speculate that long-term AST-120 administration from early CKD stage might be essential to improve patient survival after dialysis initiation. However, this contradictory result suggests that multiple factors may have a considerable impact on patient survival and also that there may be difficulties with retrospective analysis of CKD patients with heterogeneous backgrounds. Prospective trials are therefore necessary to confirm the beneficial effects of AST-120 on patient survival after dialysis initiation.

\section{Conclusions}

Although the present study is retrospective and has some limitations, our results support the evidence that AST120 treatment is associated with significant delays in the cumulative dialysis initiation rate. However, it has no effect on patient survival after dialysis initiation. A prospective randomized study may be necessary to probe survival benefit.

\section{Abbreviations}

CKD: Chronic kidney disease

BMI: Body mass index

$\mathrm{Hb}$ : Hemoglobin

sCr: Serum creatinine

BUN: Blood urea nitrogen

eGFR: Estimated glomerular filtration rate

Alb: Albumin
IP: Inorganic phosphate

Ca: Calcium

IP: Inorganic phosphorus

BP: Blood pressure

CVD: Cardiovascular disease

DM: Diabetes mellitus

Vit D: Vitamin D

ACEIs: Angiotensin-converting enzyme inhibitors

ARBs: Angiotensin receptor blockers.

\section{Conflict of Interests}

The authors declare that there is no conflict of interests.

\section{Acknowledgments}

The authors thank Mrs. Saori Araya for her invaluable help with the original data. This study was supported by the Grants-in-Aid for Scientific Research 23791737 from the Japan Society for the Promotion of Science.

\section{References}

[1] E. J. Lewis, L. G. Hunsicker, W. R. Clarke et al., "Renoprotective effect of the angiotensin-receptor antagonist irbesartan in patients with nephropathy due to type 2 diabetes," The New England Journal of Medicine, vol. 345, no. 12, pp. 851-860, 2001.

[2] "K/DOQI clinical practice guidelines for chronic kidney disease: evaluation, classification, and stratification," American Journal of Kidney Diseases, vol. 39, no. 2, supplement 1, pp. S1S266, 2002.

[3] J. T. Wright Jr., G. Bakris, T. Greene et al., "Effect of blood pressure lowering and antihypertensive drug class on progression of hypertensive kidney disease: results from the AASK trial," Journal of the American Medical Association, vol. 288, no. 19, pp. 2421-2431, 2002.

[4] F. Kanai, T. Takahama, Z. Yamazaki, Y. Idezuki, and K. Koide, "Effects of oral adsorbent on experimental uremic rats," Nippon Jinzo Gakkai Shi, vol. 28, no. 9, pp. 1249-1259, 1986.

[5] T. Shoji, A. Wada, K. Inoue et al., "Prospective randomized study evaluating the efficacy of the spherical adsorptive carbon AST-120 in chronic kidney disease patients with moderate decrease in renal function," Nephron-Clinical Practice, vol. 105, no. 3, pp. c99-c107, 2007.

[6] K. Konishi, S. Nakano, S. I. Tsuda, A. Nakagawa, T. Kigoshi, and D. Koya, "AST-120 (Kremezin) initiated in early stage chronic kidney disease stunts the progression of renal dysfunction in type 2 diabetic subjects," Diabetes Research and Clinical Practice, vol. 81, no. 3, pp. 310-315, 2008.

[7] K. Koide, J. Toyama, N. Inoue et al., "Effect of an oral sorbent (AST-120) on the clinical course of uremic peak 2a in chronic renal failure," Nippon Jinzo Gakkai Shi, vol. 29, no. 9, pp. 10031011, 1987.

[8] H. Ueda, N. Shibahara, S. Takagi, T. Inoue, and Y. Katsuoka, "AST-120, an oral adsorbent, delays the initiation of dialysis in patients with chronic kidney diseases," Therapeutic Apheresis and Dialysis, vol. 11, no. 3, pp. 189-195, 2007.

[9] K. Maeda, C. Hamada, T. Hayashi et al., "Efficacy of adsorbent in delaying dialysis initiation among chronic kidney disease 
patients," Dialysis \& Transplantation, vol. 40, no. 5, pp. 212216, 2011.

[10] H. Ueda, N. Shibahara, S. Takagi, T. Inoue, and Y. Katsuoka, "AST-120 treatment in pre-dialysis period affects the prognosis in patients on hemodialysis," Renal Failure, vol. 30, no. 9, pp. 856-860, 2008.

[11] C. P. Kovesdy, E. Lerma, and K. Kalantar-Zadeh, "AST-120 for preventing progression of chronic kidney disease: what can we conclude from the available evidence?" Dialysis \& Transplantation, vol. 40, no. 5, pp. 194-195, 2011.

[12] T. Sakaguchi and T. Akizawa, "Clinical guideline review: standards for initiation of chronic dialysis," Nihon Naika Gakkai Zasshi, vol. 91, no. 5, pp. 1561-1569, 2002.

[13] S. Matsuo, E. Imai, M. Horio et al., "Revised equations for estimated GFR from serum creatinine in Japan," American Journal of Kidney Diseases, vol. 53, no. 6, pp. 982-992, 2009.

[14] G. Manjunath, M. J. Sarnak, and A. S. Levey, "Prediction equations to estimate glomerular filtration rate: an update," Current Opinion in Nephrology and Hypertension, vol. 10, no. 6, pp. 785-792, 2001.

[15] N. S. Anavekar, J. J. V. McMurray, E. J. Velazquez et al., "Relation between renal dysfunction and cardiovascular outcomes after myocardial infarction," The New England Journal of Medicine, vol. 351, no. 13, pp. 1285-1295, 2004.

[16] T. Akizawa, Y. Asano, S. Morita et al., "Effect of a carbonaceous oral adsorbent on the progression of CKD: a multicenter, randomized, controlled trial," American Journal of Kidney Diseases, vol. 54, no. 3, pp. 459-467, 2009.

[17] K. Maeda, C. Hamada, T. Hayashi et al., "Long-term effects of the oral adsorbent, AST-120, in patients with chronic renal failure," Journal of International Medical Research, vol. 37, no. 1, pp. 205-213, 2009.

[18] T. Nakamura, E. Sato, N. Fujiwara et al., "Oral adsorbent AST120 ameliorates tubular injury in chronic renal failure patients by reducing proteinuria and oxidative stress generation," Metabolism, vol. 60, no. 2, pp. 260-264, 2010.

[19] F. C. Barreto, D. V. Barreto, S. Liabeuf et al., "Serum indoxyl sulfate is associated with vascular disease and mortality in chronic kidney disease patients," Clinical Journal of the American Society of Nephrology, vol. 4, no. 10, pp. 1551-1558, 2009.

[20] M. Yu, Y. J. Kim, and D. H. Kang, "Indoxyl sulfate-induced endothelial dysfunction in patients with chronic kidney disease via an induction of oxidative stress," Clinical Journal of the American Society of Nephrology, vol. 6, no. 1, pp. 30-39, 2011.

[21] H. Shibahara and N. Shibahara, "Cardiorenal protective effect of the oral uremic toxin absorbent AST-120 in chronic heart disease patients with moderate CKD," Journal of Nephrology, vol. 23, no. 5, pp. 535-540, 2010. 


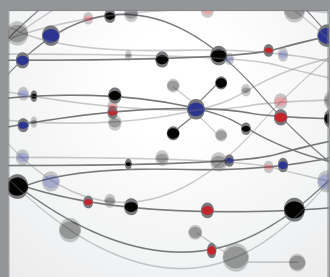

The Scientific World Journal
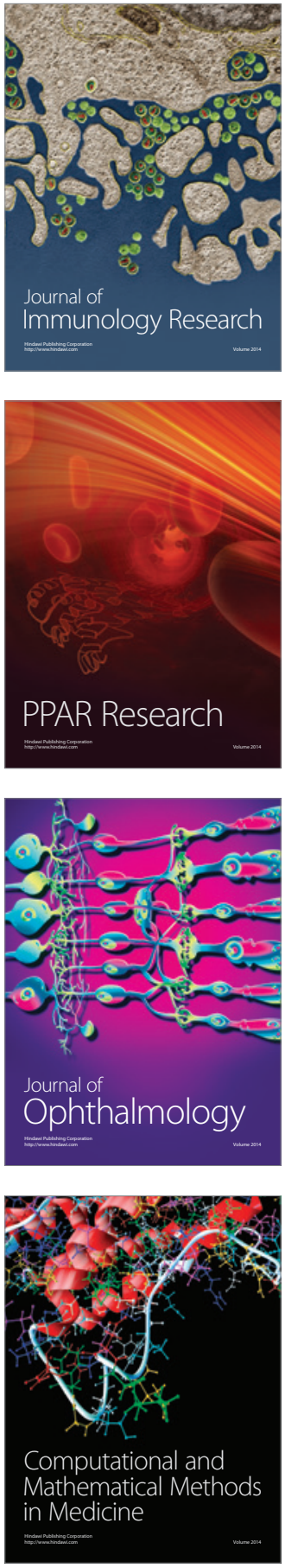

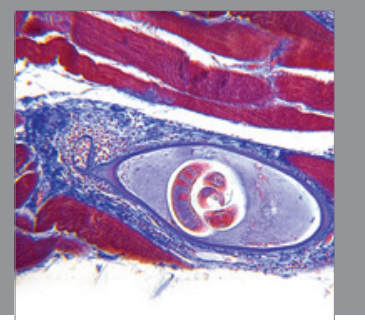

Gastroenterology

Research and Practice
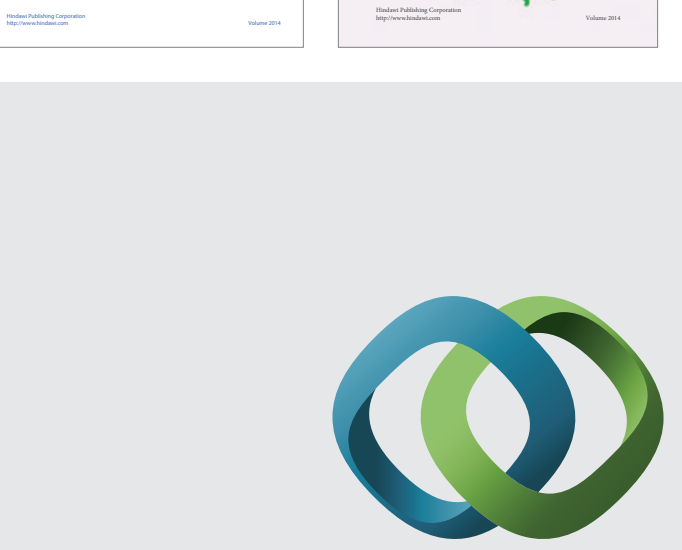

\section{Hindawi}

Submit your manuscripts at

http://www.hindawi.com
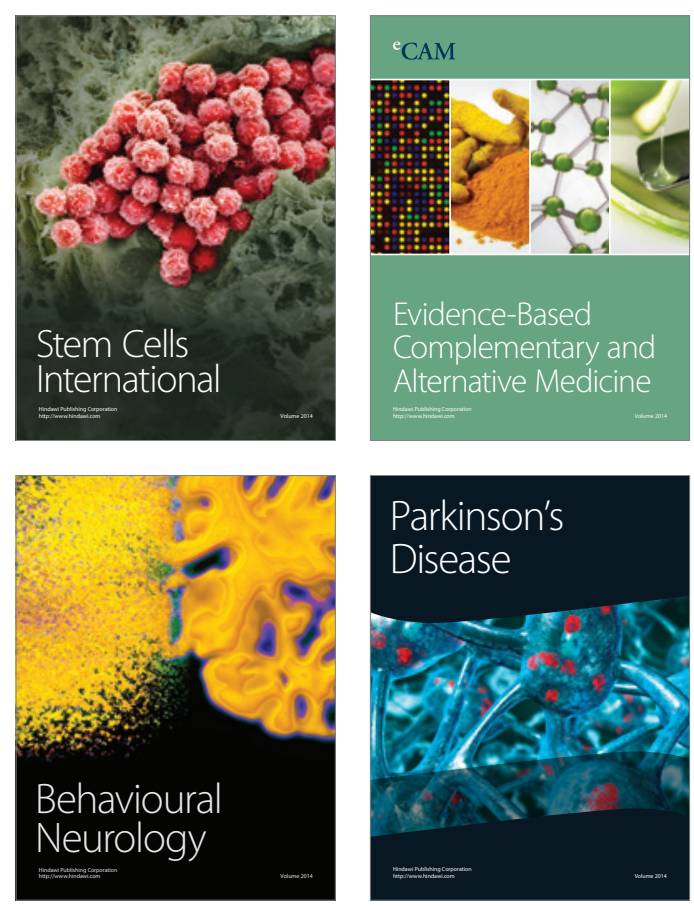

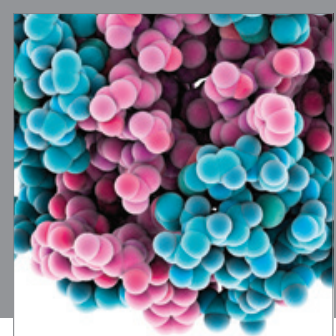

Journal of
Diabetes Research

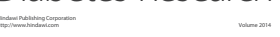

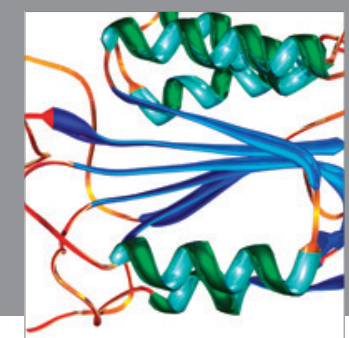

Disease Markers
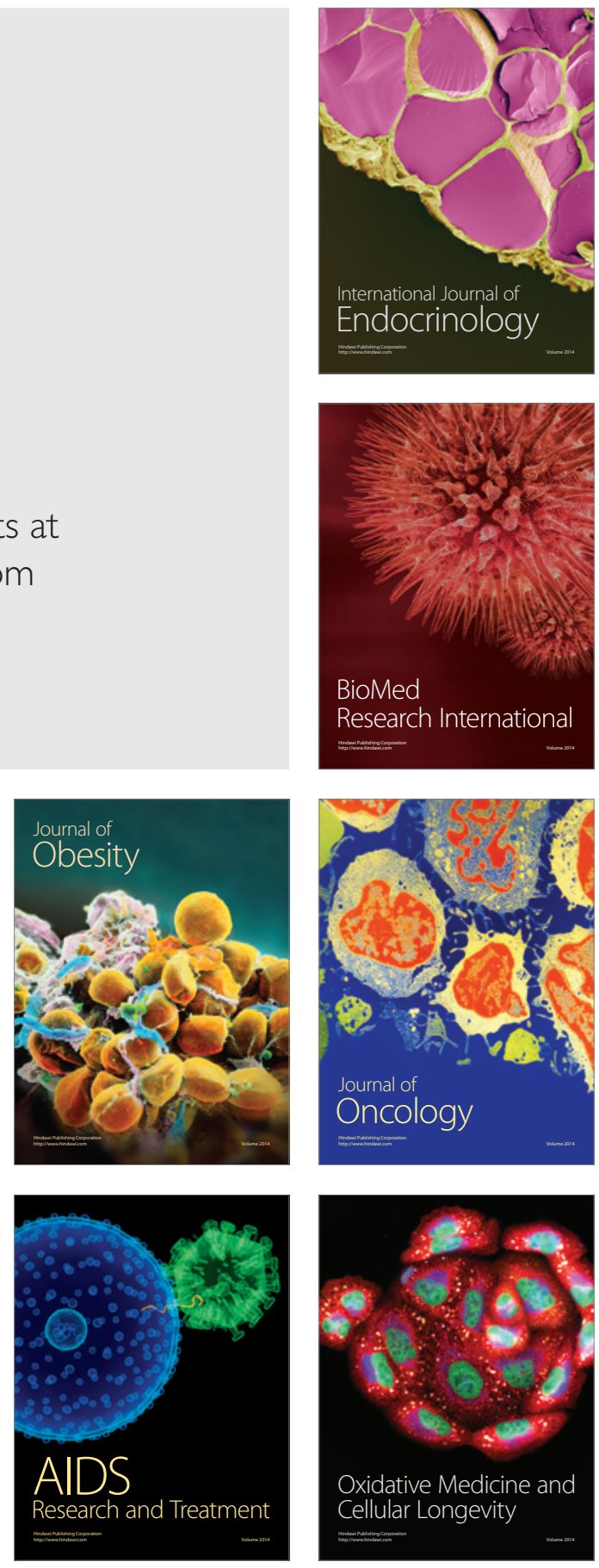